\title{
Sociocultural determinants of anticipated acceptance of pandemic influenza vaccine in Pune, India: a community survey using mixed-methods
}

\author{
Neisha Sundaram • Christian Schaetti • Leticia Grize • \\ Vidula Purohit · Saju Joseph • Christian Schindler • \\ Abhay Kudale $\cdot$ Mitchell G. Weiss
}

Received: 9 October 2015/Revised: 14 September 2016/ Accepted: 16 September 2016/Published online: 22 September 2016 (C) Swiss School of Public Health (SSPH+) 2016

\begin{abstract}
Objectives To investigate community priority and determinants of pandemic influenza vaccine acceptance in Pune, India. Community willingness to accept vaccines is often neglected in pandemic preparedness. Despite an acknowledged need, few such studies have been done in lower income countries.

Methods A cross-sectional, mixed-methods study used semi-structured explanatory model interviews to assess anticipated acceptance of nasal and injectable vaccines at different prices among 436 urban and rural residents.
\end{abstract}

Electronic supplementary material The online version of this article (doi:10.1007/s00038-016-0903-z) contains supplementary material, which is available to authorized users.

N. Sundaram · C. Schaetti · L. Grize $\cdot$ C. Schindler .

M. G. Weiss

Department of Epidemiology and Public Health, Swiss Tropical and Public Health Institute, Basel, Switzerland

N. Sundaram $(\bowtie) \cdot$ C. Schaetti $\cdot$ L. Grize $\cdot$ C. Schindler .

M. G. Weiss

University of Basel, Basel, Switzerland

e-mail: neisha.sundaram@unibas.ch

Present Address:

N. Sundaram

Saw Swee Hock School of Public Health, National University of Singapore, Singapore, Singapore

V. Purohit · S. Joseph $\cdot$ A. Kudale

Centre for Health Research and Development, The Maharashtra Association of Anthropological Sciences, Pune, India

V. Purohit · S. Joseph · A. Kudale

Department of Anthropology and Interdisciplinary School of

Health Sciences, Savitribai Phule Pune University, Pune, India
Logistic regression models identified sociocultural determinants of vaccine acceptance.

Results Over $93 \%$ anticipated acceptance at no-cost; $87.8 \%$ for INR 150 nasal vaccine; $74.1 \%$ for INR 500 and $61.7 \%$ for INR 1000 injectable vaccines. Some respondents preferred low-cost over free vaccines. Illness-related concerns about social isolation, contaminants identified as perceived causes, private-hospital or traditional-healer help seeking, and income were positively associated with anticipated acceptance. Humoral imbalances as perceived cause, home remedies for help-seeking and age were negatively associated.

Conclusions High acceptability of pandemic influenza vaccines indicates good prospects for mass vaccination. It appeared that confidence was higher in the vaccines than in the health systems delivering them. Vaccination programmes should consider sociocultural determinants influencing vaccine acceptance.

Keywords Cultural characteristics · Influenza $\cdot$ Pandemic Public participation - Social characteristics - Vaccination

\section{Introduction}

The outbreak of novel influenza A (H1N1) was declared the first influenza pandemic of the twenty-first century in June 2009. Although less severe than expected, it nevertheless imposed a substantial worldwide burden with mortality affecting children and young adults (Girard et al. 2010). Pune, located in Maharashtra, was a focus of the 2009 influenza pandemic in India, and $36 \%$ of India's H1N1-related deaths occurred in Maharashtra (Directorate General of Health Services 2010).

Vaccines are a cornerstone of influenza control. Available vaccines include intramuscularly injected inactivated 
vaccines and intranasally administered live attenuated vaccines (World Health Organization 2012). Both are considered safe and efficacious. The pandemic preparedness and response plan for India considers vaccines the "best preventive strategy to combat a pandemic" (Directorate General of Health Services 2009). However, community willingness to accept vaccines is an additional often-neglected component of effective vaccine policy and action; sociocultural features of illness that influence vaccine acceptance therefore require careful consideration.

Poor uptake of influenza vaccines during the 2009 pandemic was a problem noted worldwide (Bish et al. 2011; Poland 2010). A systematic review highlights concerns about vaccine safety and efficacy, perceived risk and seriousness of disease, social pressure, limited authoritative information, prior experience with seasonal influenza vaccine and sociodemographic characteristics associated with vaccine uptake during the 2009 pandemic (Bish et al. 2011). Notwithstanding such study of determinants of pandemic influenza vaccine acceptance, relatively little of that research has focussed on low- and middle-income countries. Acknowledged cross-national differences in pandemic response, and sociocultural influences on behaviour highlight the need for country-specific studies (Poland 2010).

To our knowledge, only two studies have examined determinants of pandemic influenza vaccine acceptance in India-one a convenience-sample study of healthcare workers (Suresh et al. 2012) and another of medical students (Suresh et al. 2011). Our study investigated the priority and determinants of vaccination intentions in the general population in Pune. The study examined the level of community interest in vaccines to prevent pandemic influenza at varying levels of cost. We also assessed sociocultural determinants of anticipated acceptance of pandemic influenza vaccines.

\section{Methods}

Study design and setting

This cross-sectional survey was conducted between July and December 2012 in two urban and two rural areas of Pune district, India. Selected urban sites comprised lowresource densely populated (slum) settlements and middleincome neighbourhoods in Pune city. Rural sites were difficult to access, remote villages in Velhe subdistrict and relatively accessible villages in Maval subdistrict.

Households were randomly selected from the local voter registry in each of the four study sites. Eligibility criteria for participants included: age range 18-65 years, residence in Pune, fluency in Marathi and ability to withstand a 45-min interview. An equal number of men and women and younger (18-45 years) and older (46-65 years) persons were interviewed. Further details of the study setting and sampling are available elsewhere (Sundaram et al. 2014).

\section{Instruments}

Interviews based on the framework of the explanatory model interview catalogue (EMIC) (Weiss 1997) are a principal tool for cultural epidemiological research (Weiss 2001). Semi-structured EMIC interviews used in this study were developed in a workshop with study investigators and public health experts (see supplementary material).

Vignettes matched for sex, site and age of the respondent, described a person with typical influenza symptoms, set in January 2010, providing a focus for questions of the interview. Coding categories derived from prior ethnographic research were used to code respondents' illness experience (categories of distress), meaning (perceived causes) and behaviour (help-seeking). Coding for responses to open questions were distinguished from elicited responses to probed categories not mentioned spontaneously. Willingness to take a vaccine to prevent swine flu, the local term commonly used for H1N1 influenza, was assessed at four different prices for both nasal and injectable vaccines: free as in the case of many immunization campaigns; low, corresponding to a subsidized price of half the market rate (INR 75 for nasal; INR 250 for injectable vaccine), medium, the market price of influenza vaccines in Pune during the 2009 pandemic (INR 150 for nasal; INR 500 for injectable vaccine) and high, an inflated price of double the market rate (INR 300 for nasal; INR 1000 for injectable vaccine), based on an inflated blackmarket rate for vaccines in short supply as they were early in the Pune pandemic. Each price level was questioned separately. Responses were recorded on a 4-point Likert scale, ranging from "yes" (clear positive response), "possibly" (qualified positive response), "uncertain" (qualified negative response) and "no" (clear negative response).

Complementary numeric and narrative data were collected in an integrated data set. Interviews were conducted in Marathi by an interviewer and a data recorder. Interviews were voice recorded with permission to enhance interview notes.

Data management and analysis

Quantitative and categorical data were double-entered using range and logic checks in Epi Info v.3.5.3 (CDC).

\section{Outcome variables}

Eight outcomes were examined: anticipated nasal and injectable influenza vaccine acceptance each at prices of 
free, low, medium, high. Each outcome variable was dichotomised as 1 (vaccine acceptance) or 0 (non-acceptance). Responses of "yes" and "possibly" were combined to represent vaccine acceptance, while "uncertain" and "no" combined represented non-acceptance.

\section{Explanatory variables}

Sociocultural variables specifying features of illness explanatory models included categories of distress, perceived causes, help seeking at home and outside, and prevention. Awareness of illness and vaccines to prevent it were also considered. Variables referring to similar aspects of a common theme were grouped. Prominence was assigned for each variable based on whether and how a respondent reported each category. Spontaneously mentioned categories received a value of 2 ; categories reported only on probing received a value of 1 , and assigned a value of 0 if not reported at all. A category identified as most important was assigned an additional value of 3. Mean values summarized the prominence of each category with a possible range of $0-5$. Sociodemographic characteristics (e.g., age, sex, occupation) were analysed as sociodemographic explanatory variables.

\section{Statistical analyses}

Fisher's exact test was used to compare proportions across different classification groups and the Cochran's Q test to compare probabilities of anticipated acceptance of free and low-cost vaccines.

To identify sociocultural and sociodemographic determinants of anticipated influenza vaccine acceptance, logistic regression analyses were performed. As a first step, bivariate associations between explanatory variables and each of the outcomes were analysed. These variables were then adjusted for sex, age and area of residence. Variables with $p<0.2$ from this analysis were selected for multivariable focal models. Focal models comprised specific variable sets (e.g., perceived causes and prevention) and provided insight into the influence of specific variables of sociocultural variable sets on vaccine acceptance. Finally, a multivariable comprehensive model containing variables from all focal models with $p<0.2$ was analysed for each outcome. Variables with $p<0.1$ were retained in the models while assessing relative quality of the model using the Akaike Information Criterion corrected for sample size (AICc). This stepwise variable reduction strategy used SAS v.9.3 (SAS Institute Inc., USA). Relative goodness of fit compared various focal and comprehensive models using $\Delta$ (AICc), representing the difference in AICc between each model and the model with the lowest AICc for each outcome. Models with lower $\Delta$ (AICc) are considered better in explaining acceptance. Regression coefficients and twotailed $p$ values are reported with statistical significance at the level of $5 \%$.

\section{Qualitative data for integrated analysis}

Narrative data helped explain the nature of explanatory variables and identified associations. Narratives were translated into English and entered in a word processor. Transcripts were managed with MAXQDA 11 (VERBI software), and were coded thematically using primarily deductive approaches. Variables from the quantitative data set were also imported into MAXQDA to select narratives of particular interest, thereby facilitating integrated analysis of qualitative and quantitative data.

\section{Results}

Among 436 completed interviews $50.7 \%$ were with women, and $51.2 \%$ were from the younger age group. Median household size was 5 persons. Median monthly household income among 342 respondents reporting their income was INR 10,000. Higher education was reported by $20.0 \%$, secondary school by $12.8 \%$ and primary school by $38.3 \%$. Incomplete primary school was reported by $7.3 \%$, and $21.6 \%$ reported no education.

Anticipated pandemic influenza vaccine acceptance

Anticipated acceptance of no-cost nasal and injectable vaccines was over $93 \%$ (Table 1), though some noted concerns:

If available free of cost, we will definitely take it. But, we need assurance that a real vaccine is being given.

We are not as educated as city people, so we are a little scared. I will make sure that the people who are vaccinating are doctors (rural woman, 27 years).

Anticipated acceptance generally declined as vaccine price increased. The urban middle-income area was an exception, where acceptance rates at the low price $(96 \%$ nasal, $95 \%$ injectable) were higher than for the free vaccine (88\% nasal, $87 \%$ injectable). A 60-year-old urban woman questioned the efficacy of a no-cost vaccine: "If they give it for free, it will not be effective, so I will not take it." Other respondents elaborated concerns about corruption and a lack of trust in government services:

If it's free then its quality is reduced. If the government is giving it, that means there is a scandal. If we instead pay 500 rupees, there is a psychological reassurance that one has taken the medicine. We do 
Table 1 Anticipated pandemic influenza vaccine acceptance at different prices among community residents: comparison of age groups and areas of residence (Pune, India 2012)

\begin{tabular}{|c|c|c|c|c|c|c|c|}
\hline \multirow[t]{3}{*}{ Vaccine price } & \multicolumn{7}{|c|}{ Anticipated vaccine acceptance $(\%)$} \\
\hline & \multirow{2}{*}{$\begin{array}{l}\text { Overall } \\
n=436\end{array}$} & \multicolumn{2}{|l|}{ Age group } & \multicolumn{4}{|c|}{ Area of residence } \\
\hline & & $\begin{array}{l}18-45 \text { years } \\
n=223\end{array}$ & $\begin{array}{l}46-65 \text { years } \\
n=213\end{array}$ & $\begin{array}{l}\text { Urban middle- } \\
\text { income } \\
n=102\end{array}$ & $\begin{array}{l}\text { Urban low- } \\
\text { resource } \\
n=113\end{array}$ & $\begin{array}{l}\text { Rural } \\
\text { remote } \\
n=108\end{array}$ & $\begin{array}{l}\text { Rural } \\
\text { accessible } \\
n=113\end{array}$ \\
\hline \multicolumn{8}{|l|}{ Nasal vaccine } \\
\hline Free (INR 0; USD 0) & 93.1 & 97.8 & $88.3 * * *$ & $88.2^{\mathrm{a}}$ & 95.6 & 97.2 & $91.2 *$ \\
\hline Low (INR 75; USD 1.4) & 91.2 & 96.8 & $85.2 * * *$ & $96.0^{\mathrm{a}}$ & 91.8 & 89.8 & 87.6 \\
\hline Medium (INR 150; USD 2.8) & 87.8 & 93.7 & $81.7 * * *$ & 92.2 & 90.3 & 87.0 & 82.3 \\
\hline High (INR 300; USD 5.6) & 82.6 & 89.7 & $75.1 * * *$ & 87.3 & 79.7 & 84.3 & 79.7 \\
\hline \multicolumn{8}{|l|}{ Injectable vaccine } \\
\hline Free (INR 0; USD 0) & 94.5 & 97.8 & $91.1 * *$ & $87.3^{\mathrm{b}}$ & 95.6 & 100.0 & $94.7 * * *$ \\
\hline Low (INR 250; USD 4.7) & 91.3 & 94.6 & $87.8 *$ & $95.1^{\mathrm{b}}$ & 87.5 & 90.7 & 92.0 \\
\hline Medium (INR 500; USD 9.3) & 74.1 & 77.1 & 70.9 & 84.3 & 70.8 & 67.6 & $74.3 *$ \\
\hline High (INR 1000; USD 18.7) & 61.7 & 70.4 & $52.6 * * *$ & 67.7 & 57.5 & 61.1 & 61.1 \\
\hline
\end{tabular}

Cochran's $Q$ test was used to compare proportions between free and low price vaccine acceptance

INR Indian Rupee, USD United States dollar, average exchange rate for 2012: 1 INR $=0.0187$ USD

Fisher's exact test was used to compare proportions across age groups and area of residence: $* p \leq 0.05, * * p \leq 0.01$, *** $p \leq 0.001$

a Cochran's $Q$ test, $p$ value $=0.05$, exact $p=0.092$

${ }^{\mathrm{b}}$ Cochran's $Q$ test, $p$ value $=0.03$, exact $p=0.057$

not trust government schemes as the vaccine may be filled with water. So it is better to take it from a private practitioner since we can afford it. (urban middle-income area, woman, 50 years).

A few respondents, however, had more faith in public services than private. Referring to experience in the 2009 pandemic, an urban man explained:

The vaccine was not being given through the government. It was available only through private providers, which is not guaranteed. We will take it if the government provides it, for that is guaranteed (65 years).

Younger age group respondents were significantly more likely to accept nasal and injectable vaccines at all prices. Older respondents were more reluctant to try new vaccines and did not want to impose a financial burden on their families.

Anticipated acceptance of the free vaccine was highest in the remote rural area and lowest in the urban middleincome area, but this pattern was reversed for the injectable vaccine priced at INR 500. Acceptance rates of men and women were similar except for the medium and high-priced injectable vaccines, where more men than women reported anticipated acceptance (medium price: women $67.4 \%$, men $80.9 \%, p=0.001$; high price: women $56.1 \%$, men $67.4 \%, p=0.018$ ).
Each respondent's pattern of acceptance at different levels of cost was analysed to determine whether acceptance was motivated by lower cost. For the nasal vaccine, $14.0 \%$ of respondents were less likely to accept as cost increased-35.6\% for the injectable vaccine. Cost was not a factor for the $76.3 \%$ (nasal) and $53.8 \%$ (injectable) who anticipated acceptance at all prices and for the $3.5 \%$ (nasal) and $1.4 \%$ (injectable) who refused the vaccine at all prices. For some, price appeared to be a counter incentive $(6.3 \%$ for nasal and $10.6 \%$ for injectable vaccines), who anticipated purchasing the high-priced vaccine despite refusing lower-priced vaccines or the free vaccine. A 46-year-old woman, who considered the injectable vaccine priced at INR 500 unaffordable, explained why she would purchase it at INR 1000 if there was high demand: "If the epidemic spreads very much, everywhere, then one would take it”.

Determinants of anticipated influenza vaccine acceptance

Multivariable focal models examined the influence of specific sets of sociocultural features of illness (i.e., perceived causes, help-seeking outside home, etc.) and sociodemographics on anticipated vaccine acceptance (Table 2). Focal models were examined for three outcomes: high-priced nasal, medium-priced injectable and high-priced 


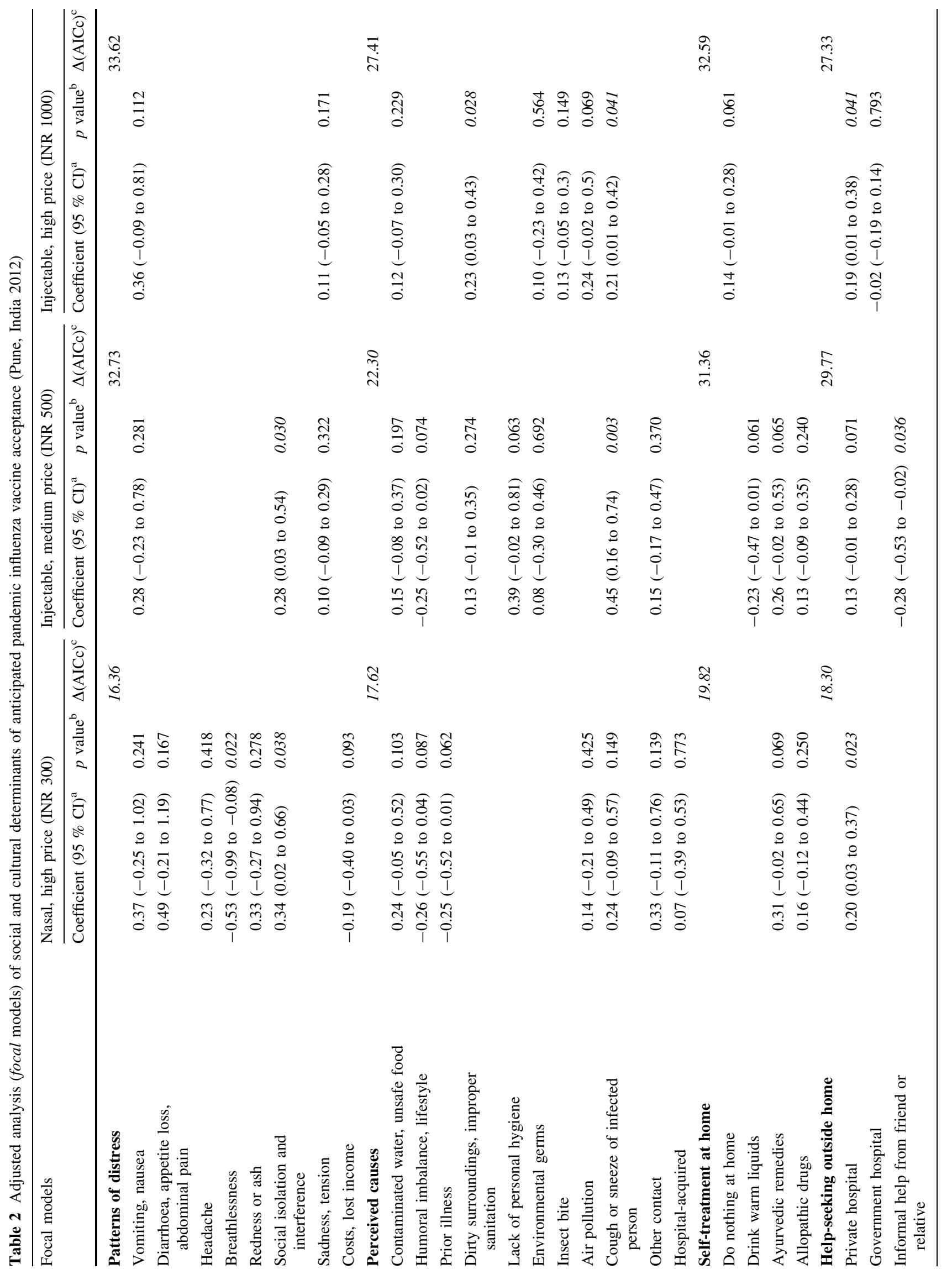




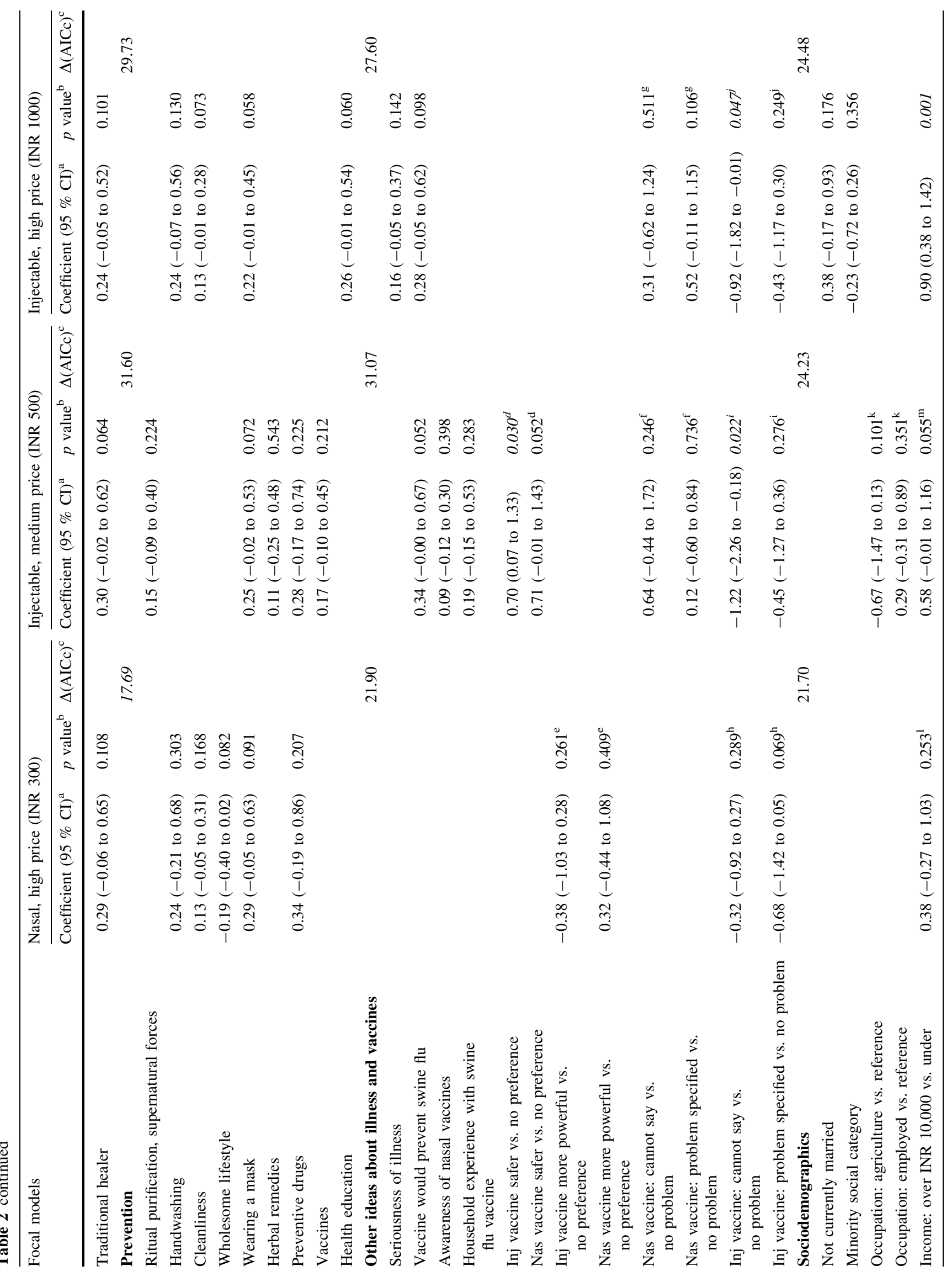




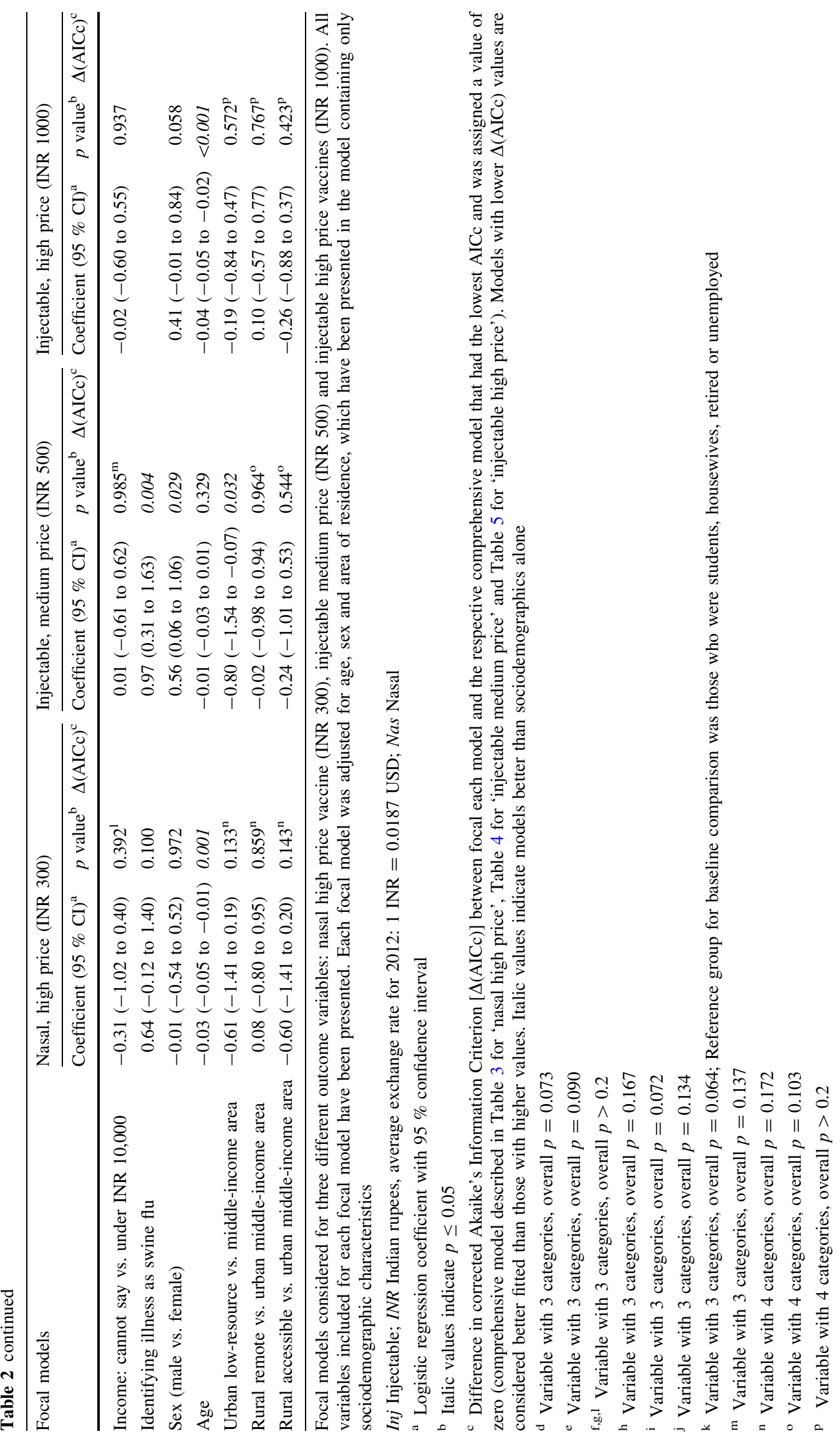


injectable vaccine acceptance. Other outcomes lacked sufficient variability for multivariable analysis. Based on $\Delta$ (AICc) values, among nasal medium price models, almost all focal models that contained sociocultural variables explained acceptance better than the exclusively sociodemographics model. But for the injectable high-price vaccine, the focal model with only sociodemographics explained acceptance better than any of the sociocultural models. For all outcomes, the comprehensive model, which combines sociocultural and sociodemographic variables from all focal models, explained acceptance best (Tables 3, 4, 5). Several variables (e.g., use of facemasks or preventive drugs in prevention, illness identification) adjusted only for sociodemographics were associated with vaccine acceptance (see Supplementary table), but did not remain significant when adjusted for additional explanatory variables.

\section{Sociodemographic features}

Income was a positive predictor of anticipated acceptance at the highest price level (Table 5), but increasing age was negatively associated for both high-priced nasal and injectable vaccines in the comprehensive models (Tables 3,5).

\section{Patterns of distress}

Reduced social contact as a troubling feature of the illness was positively associated with acceptance (Tables 3,4). Narratives explain the nature of concerns about the social impact of illness:

It will affect him socially because people tend to avoid contact with such a person. He will feel bad that he is unable to interact with people or his family due to the illness. He will feel isolated. Others would ask him to keep away. (rural man, 60 years).

Financial concerns about loss of income and cost of the illness were associated with less likely acceptance (Table 3).

\section{Perceived causes}

Perceived causes associated with more likely acceptance included consumption of contaminated water or food

Table 3 Multivariable analysis (comprehensive model) of determinants of anticipated nasal pandemic influenza vaccine acceptance at the high price (INR 300) (Pune, India 2012)

\begin{tabular}{|c|c|c|}
\hline Explanatory variables $^{\mathrm{a}}$ & Coefficient $(95 \% \mathrm{CI})^{\mathrm{b}}$ & $p$ value $^{\mathrm{c}}$ \\
\hline \multicolumn{3}{|l|}{ Patterns of distress } \\
\hline Diarrhoea, appetite loss, abdominal pain & $0.65(-0.06$ to 1.36$)$ & 0.073 \\
\hline Breathlessness & $-0.43(-0.90$ to 0.03$)$ & 0.069 \\
\hline Social isolation and interference & $0.44(0.10$ to 0.79$)$ & 0.012 \\
\hline Costs, lost income & $-0.24(-0.47$ to -0.02$)$ & 0.037 \\
\hline \multicolumn{3}{|l|}{ Perceived causes } \\
\hline Contaminated water, unsafe food & $0.30(0.0002$ to 0.59$)$ & 0.050 \\
\hline Humoral imbalance, lifestyle & $-0.36(-0.68$ to -0.05$)$ & 0.023 \\
\hline Prior illness & $-0.32(-0.62$ to -0.02$)$ & 0.037 \\
\hline \multicolumn{3}{|l|}{ Help-seeking } \\
\hline Ayurvedic remedies at home & $0.36(-0.01$ to 0.73$)$ & 0.053 \\
\hline Private hospital & $0.19(0.01$ to 0.37$)$ & 0.037 \\
\hline Traditional healers & $0.47(0.08$ to 0.85$)$ & 0.017 \\
\hline \multicolumn{3}{|l|}{ Prevention } \\
\hline Maintaining cleanliness & $0.16(-0.03$ to 0.35$)$ & 0.101 \\
\hline \multicolumn{3}{|l|}{ Sociodemographics } \\
\hline Sex (male vs. female) & $0.26(-0.31$ to 0.83$)$ & 0.376 \\
\hline Age & $-0.05(-0.07$ to -0.02$)$ & $<0.001$ \\
\hline Area of residence & & 0.148 \\
\hline Urban low-resource vs. middle-income & $-0.36(-1.23$ to 0.52$)$ & 0.424 \\
\hline Rural remote vs. urban middle-income & $-0.09(-0.97$ to 0.78$)$ & 0.837 \\
\hline Rural accessible vs. urban middle-income & $-0.86(-1.72$ to -0.003$)$ & 0.049 \\
\hline
\end{tabular}

INR Indian rupees, average exchange rate for 2012: 1 INR $=0.0187$ USD

a Variables identified in focal models $(p<0.2)$ included in comprehensive model

${ }^{b}$ Logistic regression coefficient with $95 \%$ confidence interval

${ }^{\mathrm{c}}$ Italic values indicate $p<0.05$ 
Table 4 Multivariable analysis (comprehensive model) of determinants of anticipated injectable pandemic influenza vaccine acceptance at the medium price (INR 500) (Pune, India 2012)

\begin{tabular}{|c|c|c|}
\hline Explanatory variables $^{\mathrm{a}}$ & Coefficient $(95 \% \mathrm{CI})^{\mathrm{b}}$ & $p$ value $^{\mathrm{c}}$ \\
\hline \multicolumn{3}{|l|}{ Patterns of distress } \\
\hline Social isolation and interference & $0.25(-0.01$ to 0.52$)$ & 0.058 \\
\hline \multicolumn{3}{|l|}{ Perceived causes } \\
\hline Contaminated water, unsafe food & $0.19(-0.04$ to 0.43$)$ & 0.105 \\
\hline Humoral imbalance, lifestyle & $-0.29(-0.57$ to -0.004$)$ & 0.047 \\
\hline Lack of personal hygiene & $0.43(-0.02$ to 0.88$)$ & 0.059 \\
\hline Cough or sneeze of infected person & $0.34(0.03$ to 0.64$)$ & 0.032 \\
\hline \multicolumn{3}{|l|}{ Help-seeking } \\
\hline Drink warm liquids at home & $-0.38(-0.66$ to -0.11$)$ & 0.007 \\
\hline Private hospital & $0.18(0.02$ to 0.33$)$ & 0.025 \\
\hline \multicolumn{3}{|l|}{ Other ideas about illness and vaccines } \\
\hline Illness identified as swine flu & $0.75(-0.04$ to 1.54$)$ & 0.062 \\
\hline Comparative vaccine safety & & 0.087 \\
\hline Inj vaccine: safer vs. no preference & $0.72(0.08$ to 1.37$)$ & 0.029 \\
\hline Nas vaccine: safer vs. no preference & $0.63(-0.13$ to 1.38$)$ & 0.102 \\
\hline Problems with injectable vaccine & & 0.015 \\
\hline Cannot say vs. no problem & $-0.79(-1.33$ to -0.26$)$ & 0.004 \\
\hline Problem specified vs. no problem & $-0.39(-1.14$ to 0.37$)$ & 0.318 \\
\hline \multicolumn{3}{|l|}{ Sociodemographics } \\
\hline Sex (male vs. female) & $0.48(-0.02$ to 0.98$)$ & 0.060 \\
\hline Age & $-0.01(-0.03$ to 0.01$)$ & 0.475 \\
\hline Area of residence & & 0.265 \\
\hline Urban low-resource vs. middle-income & $-0.74(-1.55$ to 0.07$)$ & 0.075 \\
\hline Rural remote vs. urban middle-income & $-0.73(-1.51$ to 0.06$)$ & 0.069 \\
\hline Rural accessible vs. urban middle-income & $-0.57(-1.37$ to 0.23$)$ & 0.161 \\
\hline
\end{tabular}

Inj Injectable; INR Indian rupees, average exchange rate for 2012: 1 INR = 0.0187 USD; Nas Nasal

a Variables identified in focal models $(p<0.2)$ included in comprehensive model

${ }^{\mathrm{b}}$ Logistic regression coefficient with $95 \%$ confidence interval

${ }^{c}$ Italic values indicate $p<0.05$

(Table 3), dirty surroundings or improper sanitation (Table 5) and cough or sneeze of an infected person (Table 4). Other negatively associated perceived causes included belief that the pandemic vignette illness was caused by some prior illness (Table 3 ) or by traditional medical ideas about a humoral imbalance or unhealthy lifestyle (Tables 3, 4).

\section{Help-seeking}

Private hospital help-seeking preference was positively associated with acceptance in all comprehensive models. Reported help-seeking from traditional healers was also positively associated (Table 3 ). Narratives indicate that traditional healers (ayurvedic doctors or herbalists) were usually mentioned as a source of help second to allopathic doctors. A reported priority of home-remedies, such as drinking warm liquids (Table 4), was negatively associated.

\section{Other ideas about vaccines}

Greater regard for safety of injectable compared with nasal vaccines was positively associated with injectable vaccine acceptance (Table 4). Interestingly, those who identified particular problems with the injectable vaccine were no less likely to anticipate acceptance than those who asserted there were "no problems", but those who could not say whether there were problems were less likely to anticipate acceptance (Tables 4, 5).

\section{Discussion}

Motivated by interest in pandemic preparedness, this study focussed on user determinants of vaccine effectiveness. Our findings indicate higher rates of community acceptance in Pune (over $93 \%$ ) than other acceptance studies for 
Table 5 Multivariable analysis (comprehensive model) of determinants of anticipated injectable pandemic influenza vaccine acceptance at the high price (INR 1000) (Pune, India 2012)

\begin{tabular}{|c|c|c|}
\hline Explanatory variables $^{\mathrm{a}}$ & Coefficient $(95 \% \mathrm{CI})^{\mathrm{b}}$ & $p$ value $^{\mathrm{c}}$ \\
\hline \multicolumn{3}{|l|}{ Perceived causes } \\
\hline Dirty surroundings, improper sanitation & $0.26(0.05$ to 0.47$)$ & 0.014 \\
\hline \multicolumn{3}{|l|}{ Help-seeking } \\
\hline Do nothing at home (go directly to hospital) & $0.13(-0.03$ to 0.28$)$ & 0.101 \\
\hline Private hospital & $0.26(0.12$ to 0.40$)$ & $<0.001$ \\
\hline \multicolumn{3}{|l|}{ Other ideas about illness and vaccines } \\
\hline Problems with injectable vaccine & & 0.003 \\
\hline Cannot say vs. no problem & $-0.83(-1.31$ to -0.34$)$ & 0.001 \\
\hline Problem specified vs. no problem & $-0.06(-0.72$ to 0.61$)$ & 0.871 \\
\hline \multicolumn{3}{|l|}{ Sociodemographics } \\
\hline Income under INR 10,000/month (ref) & & 0.002 \\
\hline Over INR 10,000 vs. under & $0.98(0.44$ to 1.51$)$ & $<0.001$ \\
\hline Cannot say vs. under INR 10,000 & $0.17(-0.43$ to 0.76$)$ & 0.579 \\
\hline Sex (male vs. female) & $0.28(-0.17$ to 0.73$)$ & 0.222 \\
\hline Age & $-0.04(-0.05$ to -0.02$)$ & $<0.001$ \\
\hline Area of residence & & 0.726 \\
\hline Urban low-resource vs. middle-income & $0.03(-0.65$ to 0.7$)$ & 0.941 \\
\hline Rural remote vs. urban middle-income & $0.23(-0.45$ to 0.92$)$ & 0.506 \\
\hline Rural accessible vs. urban middle-income & $-0.15(-0.80$ to 0.51$)$ & 0.662 \\
\hline
\end{tabular}

INR Indian rupees, average exchange rate for 2012: 1 INR $=0.0187$ USD

${ }^{a}$ Variables identified in focal models $(p<0.2)$ included in comprehensive model

${ }^{\mathrm{b}}$ Logistic regression coefficient with $95 \%$ confidence interval

${ }^{c}$ Italic values indicate $p<0.05$

no-cost vaccines in Hong Kong (45 \%) (Lau et al. 2009), United States (50\%) (Maurer et al. 2009), Australia (54\%) (Seale et al. 2010), and Malaysia (70\%) (Wong and Sam 2010). During the 2009 pandemic, vaccines were not actively made available to the general population in India. Access to vaccines was a limiting factor in Pune, even though it was a pandemic hot spot. It was also home of a vaccine manufacturer that produced the nasal influenza vaccine later in the course of the pandemic, enabled by a WHO technology transfer initiative (World Health Organization 2008). Access as a limiting factor distinguishes India from many higher-income settings where more vaccine hesitancy and less confidence are substantial. Findings suggest good prospects for community acceptance of influenza vaccines in Pune for pandemic control.

Community willingness to purchase a vaccine is probably a better indication of behaviour in settings where the vaccine is only provided at some cost. Lower anticipated acceptance at higher prices indicates that at some level cost is clearly a barrier, particularly for women and persons from remote rural areas. Subsidies would therefore be needed to implement mass vaccination for pandemic control. Although concerns about the epidemic increased the priority for purchasing high-priced vaccines, whether or not people would actually be able to afford them is another matter, as field experience suggests.

Contrary to expectations, free vaccines were less valued by some respondents, mostly in the urban middle-income area, than those with a cost. This resulted from an explicitly stated lack of trust in government services and scepticism about the quality of a free vaccine. Other research has noted that people often overreact to free products as though zero price meant not only no cost, but also an increased appeal of the product (Shampanier et al. 2007). We found the opposite phenomenon as some respondents were suspicious of the quality of a no-cost vaccine. Furthermore, confidence in health systems' capacity to deliver the vaccine may be lower than confidence in the vaccine itself. Larson (2015) found that among persons reporting a lack of confidence in immunization services, persons from India had relatively low vaccine hesitancy, compared to other countries. This context-specific finding of confidence in the vaccine but lack of trust in a free product among segments of the population has implications for implementing an influenza vaccine programme in Pune.

Regression models that incorporated sociocultural features of illness explained influenza vaccine acceptance better than those with only sociodemographics. Although 
consideration of sociocultural determinants are therefore relevant for enhancing coverage with an affordable vaccine, for high-priced vaccines-INR 1000 in our studyconsideration of sociodemographics, particularly income, supersedes other determinants.

Although influenza is not considered a traditionally stigmatized disease, like leprosy (Barrett 2005) or AIDS (Farmer and Kleinman 1989), stigmatization of collectives has been noted (Wagner-Egger et al. 2011), which can hinder control efforts (Barrett and Brown 2008). In our study, fear of social isolation or interference with relationships positively influenced vaccine acceptance. In this sociocultural context, anticipated stigma translated into anticipated vaccine acceptance.

Perceived causes influence vaccination intentions and should be assessed while planning vaccination initiatives. Among perceived causes, those related to contaminants were positively associated with vaccine acceptance, including causes with varying degrees of relevance (e.g., cough or sneeze of infected persons, contaminated food and unclean surroundings). Those identifying perceived causes unrelated to contaminants, such as cultural ideas about heat or cold in the body resulting in humoral imbalances were less likely to consider purchasing a vaccine. Efforts to increase awareness of relevant biomedical causes for influenza may thus promote vaccine uptake.

A help-seeking preference for traditional healers, however, was either unrelated or positively related to vaccine acceptance in our comprehensive models. Such traditional healers are viewed as complementary rather than competing sources of help. This may be partly explained by the fact that the Ministry of Health and Family Welfare of India (MOHFW) promoted ayurvedic interventions for prevention and treatment of H1N1 influenza alongside recommendations to visit hospitals (National Informatics Center 2012; TNN 2010). Our finding shows that acknowledging a role for healthcare providers of recognized complementary health systems is consistent with vaccine implementation.

Reporting of private hospitals to seek care may be an indication of financial ability, as these are typically more expensive than public services, or may be an indication of priority for managing influenza, as private services are perceived to provide better quality. The prominence of symptomatic relief through home remedies may have indicated a less serious condition, discouraging acceptance of the vaccine costing INR 500.

Relatively minor problems with the vaccine, such as pain or fever, were spontaneously identified by respondents. Such problems did not negatively influence anticipated acceptance, but reporting uncertainty about vaccine problems did. "Cannot say" may be a cultural euphemism for some respondents indicating understated concerns that may discourage vaccine acceptance.

In contrast to studies among the general public in the United Kingdom (Myers and Goodwin 2011) and US (Maurer et al. 2009), we found older respondents were less likely than younger persons to report intent take pandemic influenza vaccines. This generational difference is notable insofar as younger adults may be recognized as early adopters in the context of a pandemic vaccination campaign.

\section{Context of vaccine policy}

H1N1 influenza, now considered a seasonal strain, caused approximately 35,000 cases in India from January to April 2015 (Press Trust of India 2015). National control strategies have focussed on treatment with antivirals and nonpharmaceutical interventions such as handwashing, and cough etiquette (Ministry of Health \& Family Welfare 2015b). MOHFW recommends vaccination for healthcare workers, but not the public (Ministry of Health \& Family Welfare 2015a), and there is no specific mention of vaccination recommendations for other high-risk groups; such policy does not acknowledge higher mortality for people with comorbidities (World Health Organization 2015). Articles by experts have lamented the lack of clearly defined national vaccine policy for the public as a major challenge for influenza control in India (John 2015; Koul and Bali 2015). The state government of Maharashtra, however, has recently implemented a policy to vaccinate high-risk groups including pregnant women against H1N1 influenza, providing them with a no-cost vaccine in government clinics (Isalkar 2015).

Literature from other countries suggests previous experience with seasonal influenza vaccination is a major determinant of pandemic vaccine uptake (Maurer et al. 2009; Seale et al. 2010). Thus, implementation of policy for seasonal influenza vaccination in India for high-risk groups would not only decrease morbidity and mortality but also contribute to pandemic preparedness by ensuring adequate infrastructure for manufacturing influenza vaccine (Dhere et al. 2011), and improving vaccine uptake in the event of a pandemic.

\section{Limitations}

Data collection began 2 years after the pandemic potentially contributing to recall bias. Ongoing outbreaks of H1N1 influenza nevertheless may have preserved public memory of the illness. Like other studies of the influence of sociocultural factors on behaviour, our findings are context-specific, and caution is advisable in generalizing for other populations. 
Actual vaccine acceptance rates are usually lower than anticipated rates, and it was not possible to estimate the relationship between anticipated and actual uptake in this study, inasmuch as there was no public mass vaccination campaign during the 2009 pandemic in Pune. Anticipated rates are at best a guide and indication of community priority rather than a perfect prediction. Among our study respondents, $8.3 \%$ reported having personally received the vaccine during the 2009 influenza pandemic (Sundaram et al. 2015). Although this is much lower than anticipated acceptance noted among the same study respondents, it must be noted that these individuals had taken the vaccine through their own personal initiative, often at a relatively high price from private providers. The impact of limited access and the absence of an officially organized vaccination campaign with clear recommendations limited coverage. Our analysis of the high price was therefore of particular interest as an indication of confidence and commitment. Although expected to inform uptake, further research is needed to clarify the relationship between anticipated acceptance and actual uptake.

\section{Conclusion}

A strategic objective of the Global Vaccine Action Plan, approved by the World Health Assembly in 2012, emphasises that "Individuals and communities understand the value of vaccines and demand immunization as both their right and responsibility" (World Health Organization 2013). The plan further envisages that generating community and individual demand would reinforce country commitment to vaccines. Our findings indicating community confidence in pandemic influenza vaccines in a seriously affected district of India suggest good prospects both for vaccine strategies in planning for pandemic preparedness, and for broader consideration in government planning for influenza control.

Our cultural epidemiological study clarified sociocultural determinants of anticipated pandemic influenza vaccine acceptance in Pune and identified practical suggestions for effective vaccine implementation. Identified associations were explained by complementary narratives employing an integrated design in a mixed-methods approach. Our study has addressed a shortcoming in the vaccine hesitancy literature by explicitly integrating quantitative and qualitative methods (Cobos Munoz et al. 2015). By assessing vaccine confidence and determinants of anticipated use, this study highlights the relevance of sociocultural determinants and community study for influenza vaccine policy and implementation.

Acknowledgments We thank community respondents for their participation and study interviewers for their diligence and commitment.

\section{Compliance with ethical standards}

Conflict of interest The authors declare that they have no conflict of interest.

Ethical statement The Institutional Ethics Committee of the Maharashtra Association of Anthropological Sciences, Ethics Commission of Basel and WHO Research Ethics Review Committee provided ethical approval for this study. No incentives were given to participants and written informed consent was obtained prior to interview.

Funding This study was supported by the World Health Organization, Switzerland, which is thankfully acknowledged.

\section{References}

Barrett R (2005) Self-mortification and the stigma of leprosy in northern India. Med Anthropol Q 19:216-230

Barrett R, Brown PJ (2008) Stigma in the time of influenza: social and institutional responses to pandemic emergencies. J Infect Dis 197(Suppl 1):S34-S37

Bish A, Yardley L, Nicoll A, Michie S (2011) Factors associated with uptake of vaccination against pandemic influenza: a systematic review. Vaccine 29:6472-6484

Cobos Munoz D, Monzon LL, Bosch-Capblanch X (2015) Exposing concerns about vaccination in low- and middle-income countries: a systematic review. Int J Public Health 60:767-780

Dhere R, Yeolekar L, Kulkarni P, Menon R, Vaidya V, Ganguly M, Tyagi P, Barde P, Jadhav S (2011) A pandemic influenza vaccine in India: from strain to sale within 12 months. Vaccine 29(Suppl 1):A16-A21

Directorate General of Health Services (2009) Draft action plan: Pandemic preparedness and response for managing novel influenza a H1N1 (or that arising any other novel strain of influenza). Ministry of Health and Family Welfare, Government of India. http://www.delhi.gov.in/wps/wcm/connect/ffa0498040 6954a5b451bc34262e20fd/Pandeimc+Preparedness + plan-5-6. doc?MOD=AJPERES. Accessed 10 May 2015

Directorate General of Health Services (2010) Situational updatesinfluenza A H1N1 (29 Aug 2010). Ministry of Health \& Family Welfare, Government of India. http://mohfw-h1n1.nic.in/ August2010.html. Accessed 20 Feb 2014

Farmer P, Kleinman A (1989) AIDS as human suffering. Daedalus 118:135-160

Girard MP, Tam JS, Assossou OM, Kieny MP (2010) The 2009 A (H1N1) influenza virus pandemic: a review. Vaccine 28:4895-4902

Isalkar, Umesh (17 July 2015) Swine flu vaccine for pregnant women in Maharashtra. The Times of India. http://timesofindia. indiatimes.com/india/Swine-flu-vaccine-for-pregnant-women-inMaharashtra/articleshow/48106045.cms. Accessed 8 Oct 2015

John TJ (2015) Swine flu surprises. Econ Political Wkl 50:24-26

Koul PA, Bali NK (2015) Influenza vaccination in India: challenges for universal adoption. Vaccine. doi:10.1016/j.vaccine.2015.07. 021

Larson HJ, Schulz WS, Tucker JD, Smith DM (2015) Measuring vaccine confidence: introducing a global vaccine confidence index. PLoS Curr, 7

Lau JT, Yeung NC, Choi KC, Cheng MY, Tsui HY, Griffiths S (2009) Acceptability of $\mathrm{A} / \mathrm{H} 1 \mathrm{~N} 1$ vaccination during pandemic phase of influenza $\mathrm{A} / \mathrm{H} 1 \mathrm{~N} 1$ in Hong Kong: population based cross sectional survey. BMJ 339:b4164 
Maurer J, Harris KM, Parker A, Lurie N (2009) Does receipt of seasonal influenza vaccine predict intention to receive novel H1N1 vaccine: evidence from a nationally representative survey of U.S. adults. Vaccine 27:5732-5734

Ministry of Health \& Family Welfare (2015a) Swine Flu-H1N1 (Seasonal Influenza): Action taken by Government of India. Government of India. http://mohfw.gov.in/index4.php?lang= $1 \&$ level $=0 \&$ linkid $=372 \&$ lid $=3066$. Accessed 8 Oct 2015

Ministry of Health \& Family Welfare (2015b) Swine flu-H1N1 (Seasonal Influenza): do's and dont's. Government of India. http://mohfw.gov.in/showfile.php?lid=3078. Accessed 8 Oct 2015

Myers LB, Goodwin R (2011) Determinants of adults' intention to vaccinate against pandemic swine flu. BMC Public Health 11:15

National Informatics Center (2012) Health care for common diseases: influenza A (H1N1). Government of India. http://archive.india. gov.in/citizen/health/health.php?id=7. Accessed 24 Apr 2015

Poland GA (2010) The 2009-2010 influenza pandemic: effects on pandemic and seasonal vaccine uptake and lessons learned for seasonal vaccination campaigns. Vaccine 28(Suppl 4):D3-D13

Press Trust of India (2015) Swine flu death toll reaches 2123. The Indian express. http://indianexpress.com/article/india/indiaothers/swine-flu-death-toll-touches-2123/. Accessed 24 Apr 2015

Seale H, Heywood AE, McLaws ML, Ward KF, Lowbridge CP, Van D, MacIntyre CR (2010) Why do I need it? I am not at risk! Public perceptions towards the pandemic (H1N1) 2009 vaccine. BMC Infect Dis 10:99

Shampanier K, Mazar N, Ariely D (2007) Zero as a special price: the true value of free products. Mark Sci 26:742-757

Sundaram N, Schaetti C, Purohit V, Kudale A, Weiss MG (2014) Cultural epidemiology of pandemic influenza in urban and rural Pune, India: a cross-sectional, mixed-methods study. BMJ Open 4:e006350

Sundaram N, Purohit V, Schaetti C, Kudale A, Joseph S, Weiss MG (2015) Community awareness, use and preference for pandemic influenza vaccines in Pune, India. Hum Vaccin Immunother 11:2376-2388

Suresh PS, Thejaswini V, Rajan T (2011) Factors associated with 2009 pandemic influenza A (H1N1) vaccination acceptance among university students from India during the post-pandemic phase. BMC Infect Dis 11:205

Suresh PS, Rajan T, Thejaswini V, Rajeshkannan R (2012) Psychosocial determinants of 2009 pandemic influenza A (H1N1) vaccine acceptability among Indian health-care workers during the post-pandemic phase. J Infect 65:90-93

TNN (2010) Alternative medicine may help in preventing swine flu: MOHFW. The Times of India. http://timesofindia.indiatimes.com/ city/lucknow/Alternative-medicine-may-help-in-preventing-swineflu-MOHFW/articleshow/6193466.cms. Accessed 24 Apr 2015

Wagner-Egger P, Bangerter A, Gilles I, Green E, Rigaud D, Krings F, Staerkle C, Clemence A (2011) Lay perceptions of collectives at the outbreak of the H1N1 epidemic: heroes, villains and victims. Public Underst Sci 20:461-476

Weiss MG (1997) Explanatory model interview catalogue (EMIC): framework for comparative study of illness. Transcult Psychiatry $34: 235-263$

Weiss MG (2001) Cultural epidemiology: an introduction and overview. Anthropol Med 8:5-29

Wong LP, Sam IC (2010) Factors influencing the uptake of 2009 H1N1 influenza vaccine in a multiethnic Asian population. Vaccine 28:4499-4505

World Health Organization (2008) Meeting with international partners on influenza vaccine technology transfer to developing country vaccine manufacturers: 29 to 30 October 2007 . WHO/ IVB/08.09 http://whqlibdoc.who.int/hq/2008/WHO_IVB_08.09_ eng.pdf. Accessed 25 Apr 2015

World Health Organization (2012) Vaccines against influenza WHO position paper-November 2012. Wkly Epidemiol Rec 87:461-476

World Health Organization (2013) Global vaccine action plan 2011-2020. WHO Press. http://www.who.int/immunization/ global_vaccine_action_plan/GVAP_doc_2011_2020/en/. Accessed 15 Jul 2015

World Health Organization (2015) Influenza update: update number 232. http://www.who.int/influenza/surveillance_monitoring/ updates/2015_03_09_surveillance_update_232.pdf. Accessed 25 Apr 2015 